Proc. XII Int. School on Theoretical Physics — Symmetry and Structural Properties of Condensed Matter

\title{
Absorption of Twisted and Linearly Polarized Light in Graphene with Rashba Spin-Orbit Interaction
}

\author{
M. INGLOT* AND V.K. DUGAEV \\ Department of Physics and Medical Engineering, Rzeszów University of Technology, \\ al. Powstańców Warszawy 6, 35-959 Rzeszów, Poland
}

\begin{abstract}
Theoretical analysis of the electron excitations in graphene on substrate by twisted, linear and circular polarization light is presented. We use a model of graphene with constant Rashba spin-orbit interaction. In this case, the band structure of electrons includes four energy bands. The main objective of this work is to compare light absorptions in graphene for different kinds of light, namely, twisted (with nonzero orbital angular momentum) and linear polarized light. The orbital angular momentum light is characterized by some parameters $q$ and $\ell$, which can modify the response, while for the linear polarization, the absorption is modified only in the region determined by the Rashba spin-orbit coupling $\alpha$.
\end{abstract}

DOI: 10.12693/APhysPolA.132.193

PACS/topics: 42.25.Bs, 78.20.Ci, 78.40.Fy, 81.05.ue

\section{Introduction}

Graphene is one of the most important materials for possible applications now. The absorption of light due to electron transitions in a single free-standing graphene layer has been already discussed. It is known that the absorption coefficient does not depend on the light frequency and is a constant determined by the constant of fine structure $\alpha_{0}$ [1]. For the applications, not only linear or circularly polarized light is of importance. It is also important to consider the electron-light interaction for the light created by a real laser beam.

Long ago Allen et al. demonstrated that the vector potential created by the laser beam can be described by the Laguerre-Gaussian distribution of amplitude [2]. The orbital angular momentum (OAM) appears when the electric field has an angular dependence of $\exp (\mathrm{i} \ell \theta)$, where $\ell$ is the azimuthal mode index. This property of light, associated with a certain type of laser beam, has been in the focus of attention in both theoretical and experimental contexts [3, 4]. Recently, many works have been devoted to the impact of OAM in low-dimensional systems like molecules, atoms [5-7], and quantum dots [8].

In this work we study the absorption coefficient in graphene on a substrate and its modification by the RSO interaction. We use a model with the vector potential $\boldsymbol{A}(\boldsymbol{r}, t)$, which describes the twisted light. The same vector potential for chosen direction of polarization vector $\varepsilon_{\sigma}$ like, for example, $y=0$ and with $q=0$ and $\ell=0$ gives us linear polarization (LP) of light and circular for $x=1$ and $y=1$. For these three different forms of the vector potential we calculate the absorption coefficient in graphene with the Rashba spin-orbit coupling.

*corresponding author; e-mail: ming@prz.edu.pl

\section{Model}

The Hamiltonian of graphene with Rashba spin-orbit interaction has the following form:

$$
\hat{H}_{0 \boldsymbol{k}}=v\left( \pm \tau_{x} k_{x}+\tau_{y} k_{y}\right)+\lambda\left( \pm \tau_{x} \sigma_{y}-\tau_{y} \sigma_{x}\right),
$$

where $\tau_{x}$ and $\tau_{y}$ are the Pauli matrices acting in the sublattice space of graphene, the signs \pm refer to the $K$ and $K^{\prime}$ Dirac points, respectively, $\lambda=\alpha / 2$ with $\alpha$ being the coupling constant of RSO interaction [9]. In our calculation we use the energy parameter $v=\hbar v_{F}$, where $v_{F} \simeq c / 300$ is the electron velocity in graphene.

The electron energy spectrum corresponding to Hamiltonian (1) consists of four energy bands in each valley $E_{n \boldsymbol{k}}$, where $n=1$ to 4 is the band index. We obtain $E_{n \boldsymbol{k}}= \pm \lambda \pm\left(v^{2} k^{2}+\lambda^{2}\right)^{1 / 2}$ with all possible combinations of signs.

We consider the light absorption in graphene taking into account various polarization of light: linear, circular, and the so-called Bessel light described by the vector potential $\boldsymbol{A}(\boldsymbol{r}, t)$ in the form proposed in Refs. [10,11] with the intensity of radiation for two different angular momenta $\ell$, like presented in Fig. 1 . In the calculations we include the influence of graphene substrate, which is manifested by the RSO interaction with coupling constant $\alpha$. In this case the graphene electron structure near $K, K^{\prime}$ Dirac points has four electron energy bands with the optical transitions between them $[12,13]$.

In order to describe the interaction of light and matter we use gauge invariant Hamiltonian with $\boldsymbol{k} \rightarrow \boldsymbol{k}+e \boldsymbol{A} / \hbar$ :

$$
\begin{aligned}
\hat{H} & =-\mathrm{i} v\left[ \pm \tau_{x}\left(\nabla_{x}-\frac{\mathrm{i} e A_{x}}{\hbar c}\right)+\tau_{y}\left(\nabla_{y}-\frac{\mathrm{i} e A_{y}}{\hbar c}\right)\right] \\
& +\lambda\left( \pm \tau_{x} \sigma_{y}-\tau_{y} \sigma_{x}\right) .
\end{aligned}
$$

The results will be presented for the electromagnetic wave describing twisted light (TL) compared to LP and circular polarization $(\mathrm{CP})$.

The vector potential of the TL beam in the Coulomb gauge is given by 


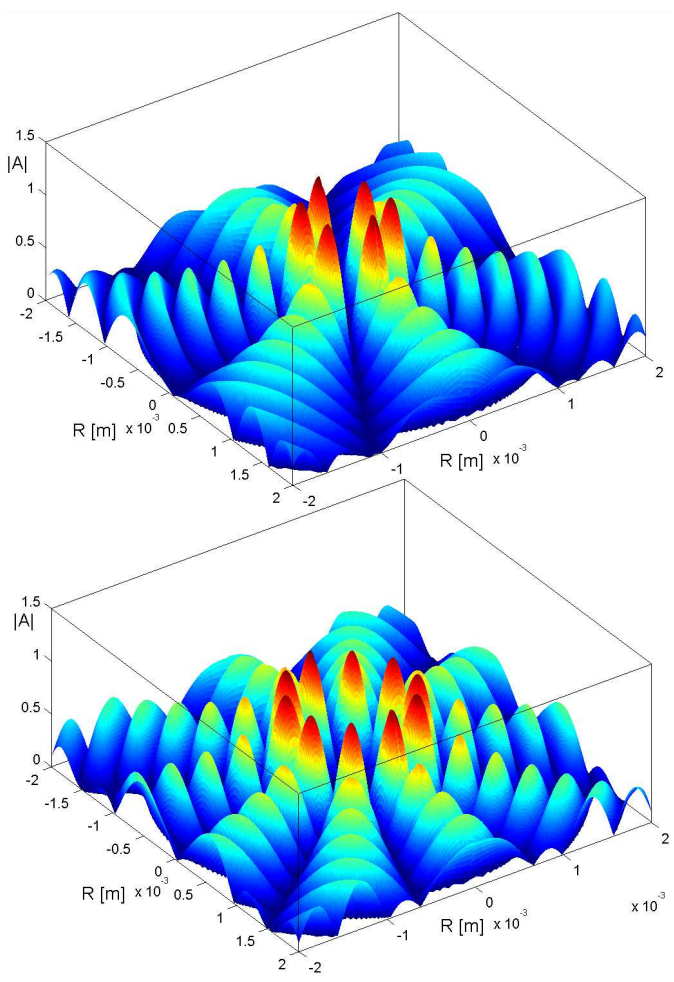

Fig. 1. Vector potential of the Bessel light $|\boldsymbol{A}|$ as a function of area position for $q=1 \times 10^{4} \mathrm{~m}^{-1}$ and angular momentum number $\ell=5$ (top) and $\ell=3$ (bottom).

$$
\begin{aligned}
& \boldsymbol{A}(\boldsymbol{r}, t)=A_{0} \mathrm{e}^{\mathrm{i}\left(q_{z} z-\omega t\right)} \\
& \quad \times\left[\boldsymbol{\varepsilon}_{\sigma} J_{\ell}(q r) \mathrm{e}^{\mathrm{i} \ell \theta}-\sigma \mathrm{i} \hat{\boldsymbol{z}} \frac{q}{q}_{z} J_{\ell+\sigma}(q r) \mathrm{e}^{\mathrm{i}(\ell+\sigma) \theta}\right]+\text { c.c. },
\end{aligned}
$$

where $\boldsymbol{\varepsilon}=\hat{\boldsymbol{x}}+\mathrm{i} \sigma \hat{\boldsymbol{y}} ; \hat{\boldsymbol{x}}, \hat{\boldsymbol{y}}, \hat{\boldsymbol{z}}$ are the unit polarization vectors, and $\sigma= \pm 1$ corresponds to right and left CP. The radial profile of the beams is given by Bessel function $J_{\ell}(q r)$ and $J_{\ell+\sigma}(q r)$. Here we use the cylindric coordinates $\boldsymbol{r}=(\boldsymbol{r}, \theta, z)$ and $\boldsymbol{q}=\left(q, \varphi, q_{z}\right)$. The wave vector $q_{z}$ is along the motion of the electromagnetic wave, which is perpendicular to the graphene plane.

\subsection{Linear and circular polarization}

In order to find the absorption coefficient in the case of circular and linear polarization we use vector potential

$$
\boldsymbol{A}_{L C}(\boldsymbol{r}, t)=A_{0} \mathrm{e}^{\mathrm{i}\left(q_{z} z-\omega t\right)} \boldsymbol{\varepsilon}_{\sigma}+\text { c.c. },
$$

where $q=0, \ell=0, q_{z}=\omega / c$, and $\omega$ is the angular frequency of incoming light, which satisfy the relation $E_{p}=\hbar \omega=h f, f$ is the light frequency and $c$ is the speed of light. The vector potential $\boldsymbol{A}_{L C}(\boldsymbol{r}, t)$ of linear polarization can be taken with $\varepsilon_{x}=1$ and $\varepsilon_{y}=0$ while the $\mathrm{CP} \varepsilon_{x, y}=1$.

The absorption coefficient can be calculated by using the Fermi golden rule

$$
\begin{aligned}
& I_{L}(\omega)=\frac{2 \pi}{\hbar} \sum_{n n^{\prime} \boldsymbol{k}}\left|\left\langle n \boldsymbol{k}\left|\hat{H}_{A}\right| n^{\prime} \boldsymbol{k}\right\rangle\right|^{2} \\
& \quad \times \delta\left(E_{n \boldsymbol{k}}+\hbar \omega-E_{n^{\prime} \boldsymbol{k}}\right) f\left(E_{n \boldsymbol{k}}\right)\left[1-f\left(E_{n^{\prime} \boldsymbol{k}}\right)\right],
\end{aligned}
$$

where $|n \boldsymbol{k}\rangle$ is the eigenfunction of Hamiltonian (1) and the interaction with electromagnetic wave is

$$
\hat{H}_{A}=-\frac{e v_{F}}{c}(\boldsymbol{\tau} \cdot \boldsymbol{A}) .
$$

In the calculations we use the absorption coefficient per unit photon flux with energy $E_{p}=\hbar \omega Q$, where $Q$ is the number of photons. Now the relation for absorption can be presented as $I_{L}(\omega)=I_{0} \sum_{n n^{\prime} k} \tilde{I}_{L}^{n \rightarrow n^{\prime}}(\omega)=I_{0} \tilde{I}_{L}(\omega)$. Thus, $I_{L}(\omega)$ can be considered as a ratio of absorption coefficients for graphene with RSO interaction and for graphene without the Rashba interaction. In other words, $\tilde{I}_{L}(\omega)$ is the absorption coefficient normalized to that for graphene without the Rashba interaction. In this case the ration $I_{0} / Q=\pi e^{2} / \hbar c=\pi \alpha_{0}$, where $\alpha_{0}=1 / 137$ is the fine-structure constant. The constant $I_{0}$ correspond to absorption coefficient at $T=0$ of graphene without RSO interaction $\alpha=0$.

In the limit of $\hbar \omega \gg \lambda$ the absorption (5) does not depend on the frequency of light. The independent of the system parameter $I_{0}$ is defined as:

$$
I_{0}=\frac{\omega}{4}\left(\frac{e}{\hbar c}\right)^{2} A_{0}^{2},
$$

where $A_{0}^{2}=4 \pi \hbar c Q / \omega$ is the square amplitude of vector potential (4) for linear polarization.

We can also consider the polarization with $x=y=1$. This means that the light is circularly polarized. In this case the amplitude of vector potential is twice smaller than LP $A_{0}^{2}=2 \pi \hbar c Q / \omega$

\subsection{Twisted light}

For the Bessel beam with nonzero OAM the vector potential is defined by Eq. (3). Now the interband optical transitions are realized with non-conserved electrons momentum, $\boldsymbol{q}=\boldsymbol{k}-\boldsymbol{k}^{\prime}$. We take the matrix elements of optical transitions as $\left\langle n \boldsymbol{k}\left|\hat{H}_{A}\right| n^{\prime} \boldsymbol{k}^{\prime}\right\rangle$, where the eigenfunctions are $|n \boldsymbol{k}\rangle=\frac{\mathrm{e}^{\mathrm{i} \boldsymbol{k} \cdot \boldsymbol{r}}}{\sqrt{\Omega}} \chi_{n \boldsymbol{k}}$, where $\Omega$ is the sample area and the bispinor $\chi_{n \boldsymbol{k}}$ is the eigenvector of the Hamiltonian without electromagnetic field (1).

The absorption of twisted light can be presented by

$$
\begin{aligned}
& \tilde{I}_{B}(\omega)=P_{B} \sum_{n n^{\prime} \boldsymbol{k} k^{\prime}}\left|\chi_{n \boldsymbol{k}}^{\dagger} \hat{H}_{A} \chi_{n^{\prime} \boldsymbol{k}^{\prime}}\right|^{2} \delta\left(E_{n \boldsymbol{k}}+\hbar \omega-E_{n^{\prime} \boldsymbol{k}^{\prime}}\right) \\
& \quad \times\left. f\left(E_{n \boldsymbol{k}}\right)\left[1-f\left(E_{n^{\prime} \boldsymbol{k}^{\prime}}\right)\right]\right|_{\boldsymbol{k}^{\prime}=k+q} .
\end{aligned}
$$

The normalization constant for the Bessel beam is $P_{B} I_{0}=\left|A_{0}\right|^{2} 8 \pi e^{2} v_{F}^{2} /\left(\hbar c^{2} q^{4} \Omega^{2}\right)$, where the square amplitude of vector potential has the form

$$
\left|A_{0}\right|^{2}=\frac{4 c q \hbar \omega Q \Omega}{R(\boldsymbol{r} q)^{-2 \ell}\left(c^{2} q^{2}+2 \omega^{2}\right) B_{\ell}(\boldsymbol{r} q)}
$$

and $R$ is the radius of sample, whereas the dimensionless function is $B_{\ell}(\boldsymbol{r} q)=-2 \ell(\boldsymbol{r} q)^{2 \ell} J_{\ell}(\boldsymbol{r} q) J_{\ell+1}(\boldsymbol{r} q)+$ $(\boldsymbol{r} q)^{2 \ell+1}\left[J_{\ell}^{2}(\boldsymbol{r} q)+J_{\ell+1}^{2}(\boldsymbol{r} q)\right]$ with the Bessel function of the first kind $J_{\nu}(x)$.

\section{Summary and conclusion}

Our main results are presented in Figs. 2 and 3, where the absorption coefficient in graphene is presented as a function of photon energy $\hbar \omega$ in the units of RSO 


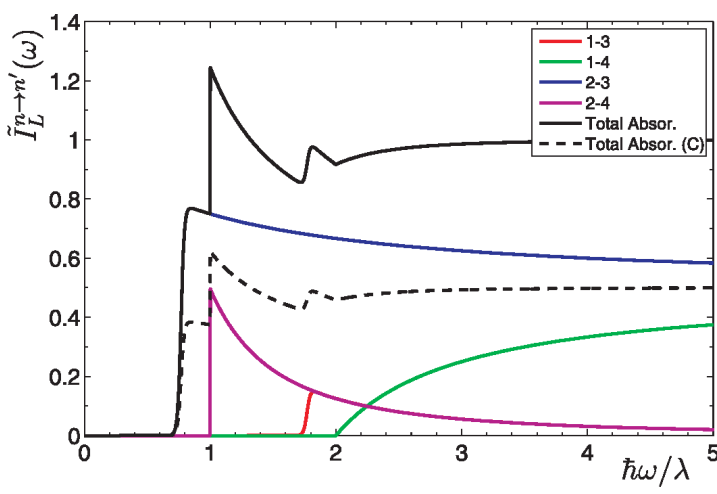

Fig. 2. Absorption coefficient of graphene with the linearly polarized light (dashed black line is CP) with Rashba spin-orbit interaction $\alpha=13 \mathrm{meV}$ and chemical potential $\mu=5 \mathrm{meV}$. Colour lines correspond to different interband optical transitions.

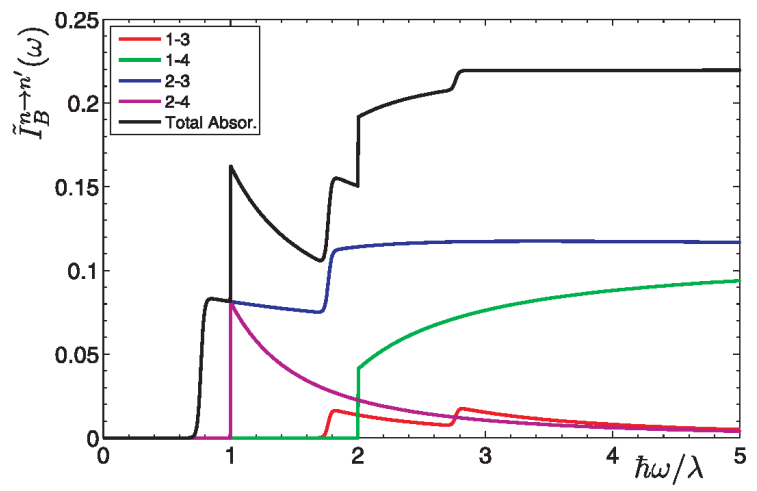

Fig. 3. Absorption coefficient of graphene with Rashba spin-orbit interaction $\alpha=13 \mathrm{meV}$ for the twisted light with $q=1 \times 10^{4} \mathrm{~m}^{-1}$ and $\ell=3$, the sample radius $R=1 \mathrm{~mm}$.

coupling $\lambda$. In contrast to the free standing graphene the absorption is not a constant over the whole range of photon energies but is strongly modified by RSO interaction generated by the substrate. The momentum of electrons is conserved for the optical interband transitions induced by linear or circular polarized light. In both cases we chose the chemical potential $\mu=5 \mathrm{meV}$ thus the first electron transition is possible from $n=2$ to $n=3$ bands
( $n$ labeling the bands in the order of increasing energy). The absorption edge is shown by blue line and is smaller than the Rashba coupling. The black line corresponds to the total absorption coefficient in graphene, whereas the solid and dashed lines are related to linear and circular polarizations, respectively. The parameters $q$ and $\ell$ strongly influence the absorption. It means that we can modify the number of absorbed photons in graphene by changing the parameters $\ell$ and $q$.

\section{Acknowledgments}

This work is supported by the National Science Center in Poland as a research project No. DEC2012/06/M/ST3/00042.

\section{References}

[1] M.I. Katsnelson, Graphene: Carbon in Two Dimensions, Cambridge Univ. Press, Cambridge 2012.

[2] L. Allen, M.W. Beijersbergen, R.J.C. Spreeuw, J.P. Woerdman, Phys. Rev. A 45, 8185 (1992).

[3] G. Spektor, A. David, G. Bartal, M. Orenstein, A. Hayat, Opt. Expr. 23, 32759 (2015).

[4] A.T. O'Neil, I. MacVicar, L. Allen, M.J. Padgett, Phys. Rev. Lett. 88, 053601 (2002).

[5] M. Babiker, C.R. Bennett, D.L. Andrews, L.C. Dávila Romero, Phys. Rev. Lett. 89, 143601 (2002).

[6] H.M. Scholz-Marggraf, S. Fritzsche, V.G. Serbo, A. Afanasev, A. Surzhykov, Phys. Rev. A 90, 013425 (2014).

[7] A.A. Peshkov, S. Fritzsche, A. Surzhykov, Phys. Rev. A 92, 043415 (2015).

[8] G.F. Quinteiro, P.I. Tamborenea, Phys. Rev. B 79, 155450 (2009).

[9] E.I. Rashba, Phys. Rev. B 79, 161409(R) (2009).

[10] R. Jáuregui, Phys. Rev. A 70, 033415 (2004).

[11] G.F. Quinteiro, P.I. Tamborenea, Phys. Rev. B 82, 125207 (2010).

[12] M. Inglot, V.K. Dugaev, E.Y. Sherman, J. Barnaś, Phys. Rev. B 89, 155411 (2014).

[13] M. Inglot, V.K. Dugaev, E.Y. Sherman, J. Barnaś, Phys. Rev. B 91, 195428 (2015). 